\title{
Is there any association between gut microbiota and type 1 diabetes? A systematic review
}

\author{
Parnian Jamshidi ${ }^{1 \dagger}$, Saba Hasanzadeh ${ }^{1 \dagger}$, Azin Tahvildari ${ }^{1 \dagger}$, Yeganeh Farsi ${ }^{1 *}$, Mahta Arbabi $^{1 *}$, João Felipe Mota ${ }^{2}$,
} Leonardo A. Sechi ${ }^{3}$ and Mohammad Javad Nasiri ${ }^{* *}$ (B)

\begin{abstract}
Introduction: Type 1 diabetes (T1D) is the second most common autoimmune disease among children. There is evidence suggesting that dysbiosis of some gut colonizing bacteria are associated with the pathogenesis of T1D. However, these studies are still controversial and a systematic review was conducted to evaluate the association between gut microbiota and T1D.
\end{abstract}

Methods: A systematic search was carried out in Medline (Via Pubmed) and Embase from January 2000 to January 2019 for all original cross-sectional, cohort, case-control or nested case-control studies investigating the association between gut microbiota and T1D.

Results: Of 568 articles identified, 26 studies met the inclusion criteria. The total population study of these articles consists of 2600 children (under 18 years old) and 189 adults. Among the included studies, 24 articles confirmed the association between gut microbiota dysbiosis and T1D. The most common bacterial alterations in T1D patients included Bacteroides spp., Streptococcus spp., Clostridium spp., Bifidobacterium spp., Prevotella spp., Staphylococcus spp., Blautia spp., Faecalibacterium spp., Roseburia spp., and Lactobacillus spp.

Conclusion: Our study showed a significant association between alterations in intestinal microbial composition and T1D; however, in some articles, it is not clear which one happens first. Investigation of altered gut microbiota can help in the early detection of T1D before seropositivity. Targeted microbiome modulation can be a novel potential therapeutic strategy.

Keywords: Type 1 diabetes, Microbiota, Dysbiosis

\section{Introduction}

Type 1 diabetes (T1D) is the second most common autoimmune disease among children. It is accompanied by many complications and has life-long morbidity [1]. The incidence of T1D is increasing universally

\footnotetext{
*Correspondence: yeganehfarsi@sbmu.ac.ir; mahtaarbabi@sbmu.ac.ir; mj.nasiri@hotmail.com

†Parnian Jamshidi, Saba Hasanzadeh and Azin Tahvildari equally first authors

${ }^{1}$ Student Research Committee, School of Medicine, Shahid Beheshti University of Medical Sciences, Tehran, Iran

${ }^{4}$ Department of Microbiology, School of Medicine, Shahid Beheshti University of Medical Sciences, Tehran, Iran

Full list of author information is available at the end of the article
}

and accounts for $5-10 \%$ of all diabetic morbidity [2]. T1D is a chronic autoimmune inflammatory process that affects insulin-producing beta cells of the pancreas, results in less insulin production [3]. Destruction of $90 \%$ of beta cells is a critical point that clinical manifestations emerge [4]. Because of the early onset of disease and chronicity, T1D is of great importance. Previous animal and human studies have shown the role of genetic factors like human leukocyte antigen (HLA) DQ and DRB in the pathogenesis of disease but recent studies propose the significant role of environmental factors such as gut colonizing bacteria [5]. Gut microbiota has an important role in the regulation of metabolism, systemic and local immunity [6]. From birth to age 3, gut 
microbiota undergoes a lot of changes and the microbiota composition of a 3-year-old child is similar to that of an adult [7]. The most important factors affecting gut microbiota include the type of delivery [8], breastfeeding [9] or bottle feeding, maternal microbiota composition, mother's diet during pregnancy and the western diet $[5,10-13]$, contact with peers, environment, and use of antibiotics [14-17]. Gut dysbiosis, an imbalance of the microbial communities, can be associated with metabolic disorders, obesity, insulin resistance, Type 2 diabetes (T2D), inflammatory bowel disease, celiac disease and immunity dysfunction [18-20]. Lately, there is evidence suggesting the correlation between dysbiosis and pathogenesis of T1D [21]. However, these studies are still controversial and need further investigation; Thus, we carried out a systematic review about the association between gut microbiota and T1D according to the Preferred Reporting Items for Systematic Reviews and Meta-Analyses statement [22].

\section{Materials and methods Search strategy}

A systematic search was carried out in Medline (Via Pubmed) and Embase from January 2000 to January 2019. Medical Subject Headings (MeSH) were "gastrointestinal microbes", "dysbiosis", "gut microbiota", "gut bacteria", "gut microbes" combined with "type 1 diabetes mellitus". Lists of references of selected articles and relevant review articles were hand-searched to identify further studies. Only studies written in English were selected.

\section{Study selection}

Two reviewers independently performed the review of titles and abstracts and chose those fitting selection criteria for full-text evaluation. Discrepancies were discussed with a third reviewer. All original cross-sectional, cohort, case-control or nested case-control studies investigating the association between gut microbiota and T1D patients were considered. The following articles were excluded: animal studies, case reports, reviews, and editorials.

\section{Data extraction}

The following variables were extracted: first author; year of publication; study duration, type of study, country/ies where the study was conducted; the number of cases with T1D; age; gender; microbiota analysis technique; modifications of intestinal microbiota and modifications of biochemical and immunological factors. Data were independently collected by two authors.

\section{Results}

The selection process of articles is shown in Fig. 1. Twenty-six articles were included and classified into 16 case-control studies [18, 21, 23-36], 6 cohort studies [37-42], 2 cross-sectional studies [5, 43] and 2 nested case-control studies $[44,45]$. Four of these studies were conducted in the USA, three in Italy, three in Finland, two in China, two in Spain and others in Netherland, Germany, Turkey, UK, Portugal, Poland, Russia, Mexico, Brazil, Australia, Czech Federation, and France. The population of these articles consists of 2600 children (under 18 years old) and 189 adults. The most applied techniques for detection and assessment of gut microbiota in stool samples where PCR, real-time quantitative PCR, 16s rRNA sequencing, microarray analysis, proteomics and quantitative cultures of stool samples (Table 1).

\section{Gut microbiota and type 1 diabetes}

Twenty-four out of twenty-six articles confirmed the association of T1D and gut microbiota dysbiosis. In one study alterations could not be attributable to T1D [23] and one of the articles is only a preliminary study and doesn't have any obvious conclusion yet [21] (Table 2). The most common bacterial alterations in T1D patients group versus healthy individuals included Bacteroides spp., Streptococcus spp., Clostridium spp., Bifidobacterium spp., Prevotella spp., Staphylococcus spp., Blautia spp., Faecalibacterium spp., Roseburia spp., and Lactobacillus spp. Details indicating the altered bacteria are shown in Table 3.

\section{The relationship between intestinal microbiota and $\mathrm{HbA1C}$, inflammatory mediators and serum zonulin level}

Some articles reported evidence of an association between $\mathrm{HbA1C}$ level and bacterial groups such as Blautia spp. count and Firmicutes:Bacteroidetes ratio (F:B ratio) [18, 33]. Murri et al. [26] in 2013 by designing a case-control study noticed the HbA1C level affected Clostridium spp. positively and F:B ratio negatively in both uni and multivariant statistical analysis. Univariate statistical analysis also showed that Bifidobacterium spp. and Lactobacillus spp. may affect HbA1C levels [26]. On the contrary, Alkanani et al. [5] reported that bacterial alterations in the case group were not associated with the HbA1C level.

There is an elevated level of TNF- $\alpha$ expression in lamina propria of intestinal biopsy in T1D patients in comparison with healthy individuals [27]. Higuchi et al. [34] reported a negative correlation between TNF plasma level and Proteobacteria and Clostridiaceae abundance. In another study, increment of Bacteroides spp. and 


\section{Search in electronic databases $(n=568)$}

Medline (via PubMed): 63

Embase: 505

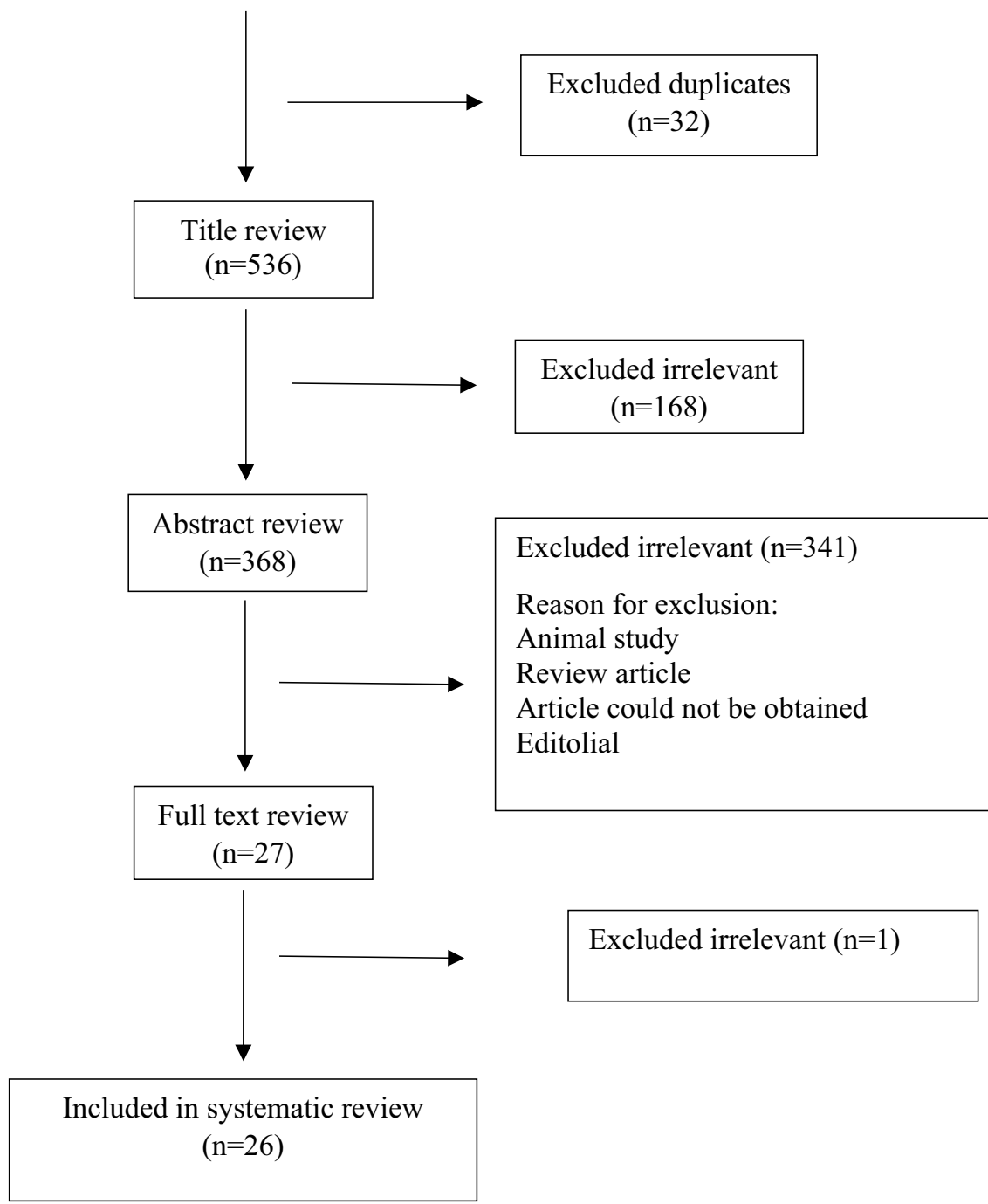

Fig. 1 Flow chart of study selection for inclusion in the systematic review

decrement of Roseburia spp. was correlated with TNF- $\alpha$ level [33].

Interleukin-6 has an important correlation with Ruminococcaceae abundance as reported by the Higuchi et al. study [34]. Increase of Bacteroides spp. and decrease of Roseburia spp. abundance is correlated with serum IL-6 level [33].

According to Leiva-Gea et al. [33] an increase in Bacteroides spp. and Veillonella spp. and decrease in Bifdobacterium spp., Roseburia spp. and Faecalibacterium spp. was associated with serum IL-1 $\beta$; in addition, an increase of Streptococcus spp. and decrease of Bifidobacterium spp. was reported related to serum IL-10 and IL-13 levels.

Serum zonulin level has a significant role in the pathogenesis of T1D. Leiva-Gea et al. [33] showed that an increase in Bacteroides spp. and Veillonella spp. and decrease in Faecalibacterium spp. and Roseburia spp. was correlated with an increased serum zonulin level. 
Table 1 Characteristics of included studies

\begin{tabular}{|c|c|c|c|c|c|c|}
\hline Authors & Year & Country & Type of study & $\begin{array}{l}\text { Study population (control } \\
\text { and case) }\end{array}$ & Age (mean) & $\begin{array}{l}\text { Microbiota analysis } \\
\text { technique }\end{array}$ \\
\hline Rozanova et al. [41] & 2002 & Russia & Cohort & $38 \mathrm{T1D}$ & 3 years & Not mentioned \\
\hline Brown et al. [23] & 2011 & Finland & Case-control & 8T1D & Children & DNA sequencing \\
\hline Giongo et al. [31] & 2011 & USA & Case-control & Control: 4, case: 4 & 5 months & $16 s$ rRNA sequencing \\
\hline Murri et al. [26] & 2013 & Spain & Case-control & Control: 16, case: 16 & 7 years & Real time quantitative PCR \\
\hline Richardson et al. [37] & 2014 & Finland & Cohort & Control: 47, case: 29 & 2 years & 16s rRNA sequencing \\
\hline de Goffau et al. [24] & 2014 & Netherlands & Case-control & Control: 27, case: 28 & 3 years & Microarray analysis \\
\hline Endesfelder et al. [38] & 2014 & Germany & Cohort & Control: 22, case: 22 & 19.5 months & 16s rRNA sequencing \\
\hline Mejia et al. [30] & 2014 & Mexico & Case-control & Control: 8, case: 21 & 12.5 years & 16s rRNA pyrosequencing \\
\hline Soyucen et al. [28] & 2014 & Turkey & Case-control & Control: 35, case: 35 & 10 years & $\begin{array}{l}\text { Quantitative cultures on } \\
\text { selective and non-selective } \\
\text { media }\end{array}$ \\
\hline Kostic et al. [39] & 2015 & Finland & Cohort & Control: 22, case: $11^{\mathrm{b}}$ & Infants & 16s rRNA sequencing \\
\hline Alkanani et al. [5] & 2015 & USA & Cross-sectional & Control: 23, case ${ }^{\text {a. }} 88$ & 11 years & 16s rRNA sequencing \\
\hline Cui et al. [18] & 2016 & China & Case-control & Control: 15, case: 15 & 11 years & $16 s$ rRNA sequencing \\
\hline Maffeis et al. [25] & 2016 & Italy & Case-control & Control: 10, case: 10 & 11 years & Semi-quantitative PCR \\
\hline Stewart et al. [29] & 2017 & UK & Case-control & Control: 10, case: 10 & 27 years & $16 s$ rRNA sequencing \\
\hline Pinto et al. [40] & 2017 & Portugal & Cohort & Control: 3 , case: 3 & 9 years & Real time quantitative PCR \\
\hline Pellegrini et al. [27] & 2017 & Italy & Case-control & Control: 35', case: 19 & 36 years & Real time quantitative PCR \\
\hline Traversi et al. [21] & 2017 & Italy & Case-control & Control: 13, case: 13 & 8 years & Real time quantitative PCR \\
\hline Gao et al. [42] & 2018 & France & Cohort & $\begin{array}{l}33 \text { genetically predisposed } \\
\text { to T1D }\end{array}$ & 1.5 years & 16s rRNA sequencing \\
\hline Vatanen et al. [44] & 2018 & USA & Nested case-control & Control: 415 , case: $368^{d}$ & 3 month & 16s rRNA sequencing \\
\hline Stewart et al. [45] & 2018 & USA & Nested case-control & 903 children & 24.5 month & 16 s rRNA sequencing \\
\hline Huang et al. [32] & 2018 & China & Case-control & Control: 10, case: 12 & 23.5 & 16s rRNA sequencing \\
\hline Gavin et al. [43] & 2018 & Australia & Cross-sectional & Control: 22, case: $69^{\mathrm{e}}$ & 10.8 & $\begin{array}{l}\text { Proteomics and } 16 \mathrm{~S} \text { rRNA } \\
\text { sequencing }\end{array}$ \\
\hline Leiva-Gea et al. [33] & 2018 & Spain & Case-control & Control: 13, case: 15 T1D & 12.6 & 165 rRNA pyrosequencing \\
\hline Higuchi et al. [34] & 2018 & Brazil & Case-control & Control: 28, case: 20 & 23.1 & 16 s rRNA sequencing \\
\hline Salamon et al. [35] & 2018 & Poland & Case-control & Control: 23 , case: 22 & 42.5 & 16s rRNA sequencing \\
\hline Cinek et al. [36] & 2018 & Czech federation & Case-control & Control: 103 , case: $73^{f}$ & 11.8 & 16s rRNA sequencing \\
\hline
\end{tabular}

a 35 new-onset patients; 21 seropositive; 32 seronegative FDRs (first degree relatives)

b Seroconverters

c 16 healthy control; 19 gut inflammatory disease as the second control

d 267 seroconverters and 101 diagnosed with T1D

e 23 recent onset type 1 diabetes; 17 islet autoantibody-positive subjects; 29 low-risk autoantibody-negative subjects

f Azerbaijan: 19, Jordan: 20, Nigeria: 14, Sudan: 20

\section{Discussion}

We reviewed 26 articles, twenty-four of them approved a straight correlation between microbiota and diabetes; however, most of them didn't clarify if microbiota induces T1D or T1D changes gut microbiome. The articles were screened according to the type of gut microbiota and correlation with T1D as explained below: one article mentioned that microbiome alteration occurs after diabetes [26], two articles studied microbiota as a therapeutic agent on T1D $[35,41]$, seven articles just showed the differences in gut microbiota of healthy and diabetic people and didn't discuss the type of relation $[21,24,29,32,34,36,40]$, finally fourteen articles suggested the exact mechanism that leads to autoimmunity by the change in gut microbiome $[5,18,25,27,28,31$, $33,37,39,42-46]$ (one article was just in the abstract form and we couldn't read the details [30]).

Different mechanisms have been suggested about the role of gut microbiota in the pathogenesis of T1D. These mechanisms are mainly derived from the 14 articles mentioned above. In more details, the following points can be noticed: 
Table 2 Association of gut microbiota and type 1 diabetes

\begin{tabular}{|c|c|c|c|c|}
\hline Authors & Year & Country & Type of study & $\begin{array}{l}\text { Association } \\
\text { between diabetes } \\
\text { and microbiome }\end{array}$ \\
\hline Rozanova et al. [41] & 2002 & Russia & Cohort & Yes \\
\hline Brown et al. [23] & 2011 & Finland & Case-control & May \\
\hline Giongo et al. [31] & 2011 & USA & Case-control & Yes \\
\hline Murri et al. [26] & 2013 & Spain & Case-control & Yes \\
\hline Richardson et al. [37] & 2014 & Finland & Cohort & Yes \\
\hline de Goffau et al. [24] & 2014 & Netherlands & Case-control & Yes \\
\hline Endesfelder et al. [38] & 2014 & Germany & Cohort & Yes \\
\hline Mejia et al. [30] & 2014 & Mexico & Case-control & Yes \\
\hline Soyucen et al. [28] & 2014 & Turkey & Case-control & Yes \\
\hline Kostic et al. [39] & 2015 & Finland & Cohort & Yes \\
\hline Alkanani et al. [5] & 2015 & USA & Cross-sectional & Yes \\
\hline Cui et al. [18] & 2016 & China & Case-control & Yes \\
\hline Maffeis et al. [25] & 2016 & Italy & Case-control & Yes \\
\hline Stewart et al. [29] & 2017 & UK & Case-control & Yes \\
\hline Pinto et al. [40] & 2017 & Portugal & Cohort & Yes \\
\hline Pellegrini et al. [27] & 2017 & Italy & Case-control & Yes \\
\hline Traversi et al. [21] & 2017 & Italy & Case-control & Not mentioned \\
\hline Gao et al. [42] & 2018 & France & Cohort & Yes \\
\hline Vatanen et al. [44] & 2018 & USA & Nested case-control & Yes \\
\hline Stewart et al. [45] & 2018 & USA & Nested case-control & Yes \\
\hline Huang et al. [32] & 2018 & China & Case-control & Yes \\
\hline Gavin et al. [43] & 2018 & Australia & Cross-sectional & Yes \\
\hline Leiva-Gea et al. [33] & 2018 & Spain & Case-control & Yes \\
\hline Higuchi et al. [34] & 2018 & Brazil & Case-control & Yes \\
\hline Salamon et al. [35] & 2018 & Poland & Case-control & Yes \\
\hline Cinek et al. [36] & 2018 & Czech federation & Case-control & Yes \\
\hline
\end{tabular}

1. In patients with T1D, some bacteria increase mucin degradation, results in reduced integrity and increased permeability of intestinal mucosa that leads to bacterial penetration [47]. The penetration of bacteria into intestinal mucosa leads to stimulation of the immune system and production of antibodies against them [47]. Cross-reaction of these antibodies and surface antigens of pancreatic beta cells, as well as $\mathrm{T}$ cell cross-reactivity results in the destruction of beta cells and formation of T1D [47].

2. Butyrate is one of the most important byproducts of microbiota metabolisms and plays an important role in colonic T-reg induction, down-regulation of proinflammatory macrophages and integrity enhancement of gut barriers through increasing mucin production $[48,49]$.

3. Zonulin is a protein that can be assumed as an important indicator of mucosal integrity and gut permeability [33]. This protein modulates intercellular junctions and macromolecular passage through them
[33]. Some bacterial groups can alter mucosal integrity by affecting zonulin; increase in Bacteroides spp. and Veillonella spp. or decrease in Faecalibacterium spp. and Roseburia spp. correlates with increased serum zonulin levels in T1D patients [33]. However, according to Leiva-Gea et al. [33] the impaired gut permeability in T1D patients can be more attributed to the binding of Veillonella to colonic crypt cells rather than change in zonulin levels. Lactate produced by Veillonella is pushed to the luminal surface and weakens tight junctions [33].

4. Gut microbiota ingests and ferment fibers and produce short-chain fatty acids (SCFA) [50-52]. SCFAs enter the blood circulation and modulate T-reg differentiation; thus autoimmunity is prevented $[5,53-55]$.

With keeping these mechanisms in mind, now we are going to discuss the known attributable mechanism of some highlighted bacteria in more details: 


\section{Table 3 Intestinal microbiota modifications}

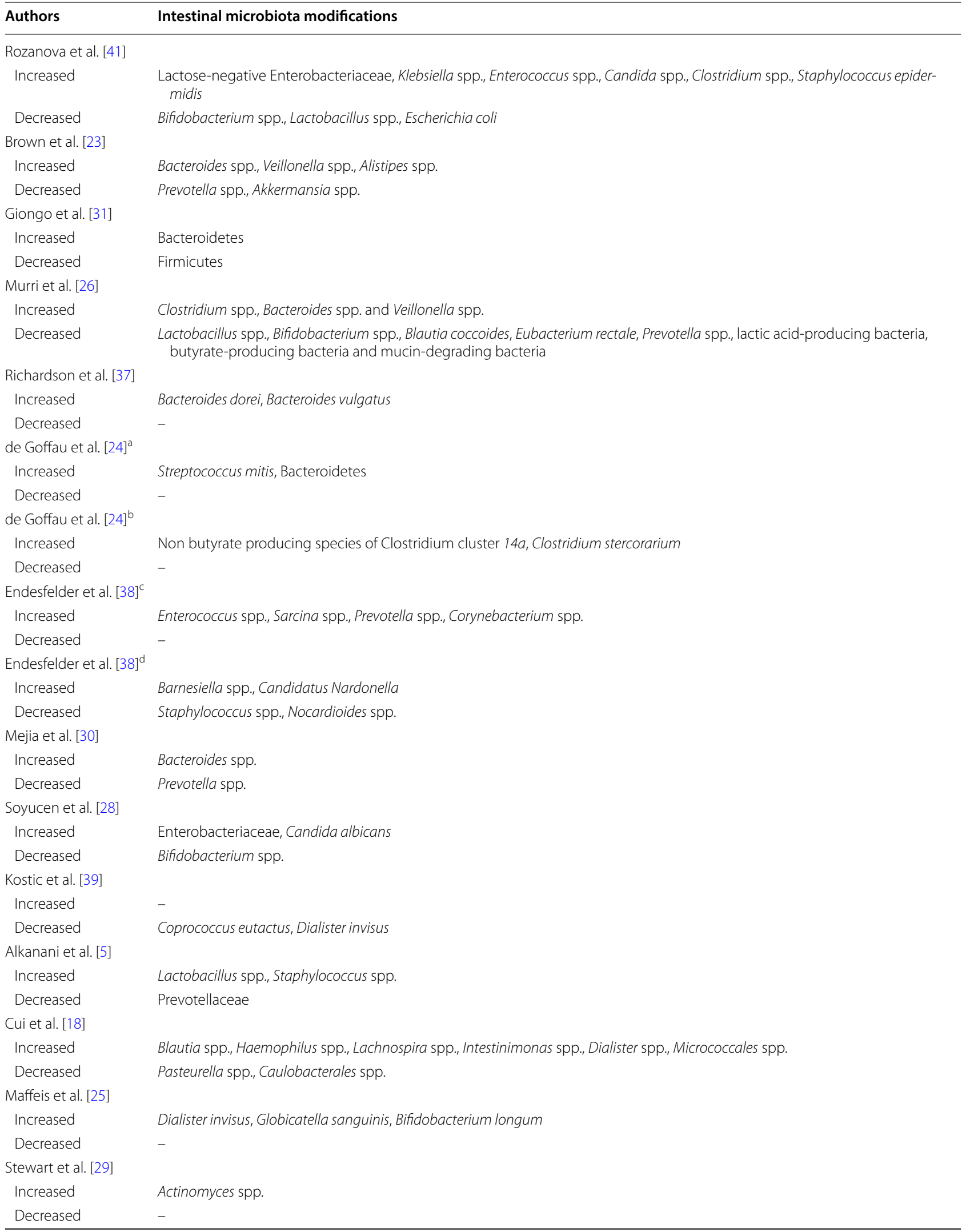


Table 3 (continued)

\begin{tabular}{|c|c|}
\hline Authors & Intestinal microbiota modifications \\
\hline \multicolumn{2}{|l|}{ Pinto et al. [40] } \\
\hline Increased & Eubacterium rectale, Faecalibacterium prausnitzzi, Bacteroides dorei, Bacteroides uniformis \\
\hline Decreased & $\begin{array}{l}\text { Collinsella aerofaciens, Coprococcus Comes, Clostridium spp., Bifidobacterium adolescentis, Bifidobacterium longum infantis, Rumi- } \\
\text { nococcus spp., Collinsella spp. }\end{array}$ \\
\hline \multicolumn{2}{|c|}{ Pellegrini et al. [27] } \\
\hline Increased & Firmicutes \\
\hline Decreased & Clostridium spp., Bacteroidetes, Proteobacteria \\
\hline \multicolumn{2}{|c|}{ Traversi et al. [21] } \\
\hline Increased & Bacteroides clarus, Alistipes obesi, Bifidobacterium longus, Methanobrevibacter Smithii \\
\hline Decreased & Bacteroides coprophilus, Bacteroides dorei, Fusicatenibacter saccharivorans, Bacteroides vulgatus, Bacteroides oleiciplenus, Firmicutes \\
\hline Gao et al. [42] & This study emphasizes on interactions between gut microbiota rather than quantitative changes \\
\hline \multicolumn{2}{|c|}{ Vatanen et al. [44] } \\
\hline Increased & Bifidobacterium pseudocatenulatum, Roseburia hominis, Alistipes shahii \\
\hline Decreased & Streptococcus thermophilus, Lactococcus lactis \\
\hline \multicolumn{2}{|c|}{ Stewart et al. [45] } \\
\hline Increased & - \\
\hline Decreased & Ruminococcus spp., Lactococcus spp., Streptococcus spp., Akkermansia spp. \\
\hline \multicolumn{2}{|c|}{ Huang et al. [32] } \\
\hline Increased & Bacteroidetes/Firmicutes ratio, Porphyromonadaceae \\
\hline Decreased & Ruminococcus spp., Veillonella spp., Phascolarctobacterium spp., Fusobacterium spp., Paenibacillaceae \\
\hline \multicolumn{2}{|c|}{ Gavin et al. [43] } \\
\hline Increased & Bacteroides spp., Prevotella spp. \\
\hline Decreased & Alistipes spp., Ruminococcus spp., Barnesiella spp., Clostridium spp., Dorea spp., Faecalibacterium Prausnitzii \\
\hline \multicolumn{2}{|c|}{ Leiva-Gea et al. [33] } \\
\hline Increased & $\begin{array}{l}\text { Bacteroides spp., Rikenellaceae, Ruminococcus spp., Veillonella spp., Enterobacteriaceae, Blautia spp., Streptococcus spp., Prevotel- } \\
\text { laceae, Sutterella spp. }\end{array}$ \\
\hline Decreased & $\begin{array}{l}\text { Bifidobacterium spp., Roseburia spp., Faecalibacterium spp., Lachnospira spp., Anaerostipes spp., Actinobacteria, Proteobacteria, } \\
\text { Firmicutes }\end{array}$ \\
\hline \multicolumn{2}{|c|}{ Higuchi et al. [34] } \\
\hline Increased & Bacteroides spp., Alistipes spp., Prevotella spp. \\
\hline Decreased & - \\
\hline \multicolumn{2}{|c|}{ Salamon et al. [35] } \\
\hline Increased & Akkermansia spp., Ruminococcus spp., Bacteroides spp., Blautia spp. \\
\hline Decreased & Lachnospira spp., Faecalibacterium spp., Bifidobacterium spp., Coprococcus spp., Collinsella spp., Dorea spp. \\
\hline \multicolumn{2}{|c|}{ Cinek et al. [36] } \\
\hline Increased & Escherichia coli \\
\hline Decreased & Eubacterium spp., Roseburia spp., Haemophilus spp., Clostridium clusters IV and XIVa \\
\hline
\end{tabular}

a In cases with $<2.9$ years

b In cases with $>2.9$ years

c Eigenvector centrality (EC) at age 0.5 years

d EC at age 2 years

Bacteroidetes, Firmicutes, Proteobacteria, and Actinobacteria were of great importance in our reviewed articles.

Genera Bacteroides and Prevotella are two important subgroups in Phylum Bacteroides that were increased in most of the T1D patient's samples and can affect gut microbial composition by several mechanisms. Succinate and acetate are the main byproducts of anaerobic metabolism in this phylum that compromise epithelial tight junctions, decrease gut mucosal integrity, block T-reg differentiation and activates inflammatory pathways $[25,32,33]$.

These bacteria also produce Glutamic acid decarboxylase (GAD) which can stimulate GAD autoimmunity by molecular mimicry $[24,32]$. 
Phylum Actinobacteria including genus Bifidobacterium is butyrate-producing taxa that have anti-inflammatory effects and augments gut barrier by cytokine modulation [33]. This bacteria also induces T-reg development that results in immune response suppression by regulation of IL-10 production [18].

The third important phylum, Firmicutes, consists of eight notable subgroups: Veillonella, Roseburia, Ruminococcus, Lactobacillus, Blautia, Streptococcus, Faecalibacterium, and Staphylococcus.

Association of T1D and Veillonella is controversy. Kostic et al. [39] reported a decrease in Veillonella in T1D patients and proposed the following mechanism: reduced level of lithocholic acid results in stimulation of gut inflammation by an increased level of reactive oxygen species, reactive nitrogen species and nuclear factor-kB (NF-kB) activity in epithelial cells. Sphingomyelin increment also inhibits NK-T cell function that prevents inflammation [39].

Ruminococcaceae are butyrate-producing taxa that were reported declined in some studies and increased in others. The mechanism of reduced Ruminococcaceae in T1D patients is the same as Veillonella reduction mechanism $[33,39]$.

Faecalibacterium and Roseburia have anti-inflammatory effects, their presence may augment gut barrier function by modulating cytokine production and butyrate synthesis $[24,33,36]$. These genera have decreased in almost all patient samples.

Genus Blautia is also butyrate-producing taxa that have declined in most of the reviewed articles and plays an important role in blood glucose regulation, lipid metabolism and regulation of T-cell differentiation $[18,33]$. There is also evidence of its increment in literature.

Genus Lactobacillus eliminates peroxidase radicals by superoxide dismutase and peroxidase enzymes thus provide a suitable condition for Bifidobacterium reproduction [28]. Lactobacillus down-modulate inflammation and previous studies have demonstrated that dendritic cells co-cultured with species of lactobacilli induce polarization of T-reg cells $[5,56,57]$.

Staphylococcaceae may stimulate the growth of Bifidobacterium, Clostridium and Bacteroides which results in augmentation of neonate's gut maturation [5, 58]. Streptococcaceae produce GAD so they have the same effects as Bacteroides [24, 59].

\section{Limitations}

Limitations of our study that complicates interpretation of results can be listed as below: various geographical areas studied have an effect on diet of patients and controls; diversity of microbiota analysis techniques; colonizing microbiome and genetic susceptibility to T1D, some studies considered HLA as a genetic predisposing factor in the selection process of case and control individuals whereas others have ignored this point; different study design (e.g. some studies noticed seropositive group and seronegative FDRs in addition to T1D patients and healthy individuals while others just compared T1D patients with healthy individuals) [5, 25, 39, 44, 45]; various statistical analysis methods and different levels of p-value significance were reported in reviewed articles, however, in this study we used only statistical significant findings from the articles included.

\section{Suggestions}

According to our study, we suggest new therapeutic and diagnostic strategies that need further clinical trials for assessing their effectiveness:

1. Use of prebiotics, probiotics and fecal microbial transplantation to modulate gut microbiome; e.g. a probiotic mixture of certain bacteria (mentioned in "Results") can reduce HbA1c level so it can be considered as a complementary strategy for T1D management.

2. In order to detect early evidence of dysbiosis and prevention of T1D progression, serial stool exams in genetically susceptible children can be done by using a specific kit that semi-quantitatively compares microbiota composition of healthy control and suspected individual. In designing the kit, Firmicutes: Bacteroidetes ratio should be considered because it has been reported decreased in all the reviewed studies.

\section{Conclusions}

Our study showed a significant association between alterations in intestinal microbial composition and T1D; however, in some articles, it is not clear which one happens first. Investigation of altered gut microbiota can help in the early detection of T1D before seropositivity against classical autoantigens. Targeted microbiome modulation can be a novel potential therapeutic strategy.

\section{Acknowledgements}

This study is related to the project No. 1397/68977 From Student Research Committee, Shahid Beheshti University of Medical Sciences, Tehran, Iran. We also appreciate "Student Research Committee" and "Research and Technology Chancellor" in Shahid Beheshti University of Medical Sciences for their financial support of this study.

\section{Authors' contributions}

All authors had made considerable contributions to the present study. All authors read and approved the final manuscript. 


\section{Funding}

Not applicable.

\section{Availability of data and materials}

All data were included.

\section{Ethics approval and consent to participate}

Not applicable.

\section{Consent for publication}

Not applicable.

\section{Competing interests}

The authors declare that they have no competing interests.

\section{Author details}

${ }^{1}$ Student Research Committee, School of Medicine, Shahid Beheshti University of Medical Sciences, Tehran, Iran. ${ }^{2}$ Clinical and Sports Nutrition Research Laboratory (LABINCE), Faculty of Nutrition, Federal University of Goiás, Goiânia, Brazil. ${ }^{3}$ Department of Biomedical Sciences, University of Sassari, Sassari, Italy. ${ }^{4}$ Department of Microbiology, School of Medicine, Shahid Beheshti University of Medical Sciences, Tehran, Iran.

Received: 22 June 2019 Accepted: 27 September 2019

Published online: 14 October 2019

\section{References}

1. Mejia-Leon ME, Barca AM. Diet, microbiota and immune system in type 1 diabetes development and evolution. Nutrients. 2015;7(11):9171-84.

2. Pettitt DJ, Talton J, Dabelea D, Divers J, Imperatore G, Lawrence JM, et al. Prevalence of diabetes in US youth in 2009: the SEARCH for diabetes in youth study. Diabetes Care. 2014;37(2):402-8.

3. Notkins $A L$, Lernmark $\AA$. Autoimmune type 1 diabetes: resolved and unresolved issues. J Clin Invest. 2001;108(9):1247-52.

4. Islam ST, Srinivasan S, Craig ME. Environmental determinants of type 1 diabetes: a role for overweight and insulin resistance. J Paediatr Child Health. 2014;50(11):874-9.

5. Alkanani AK, Hara N, Gottlieb PA, Ir D, Robertson CE, Wagner BD, et al. Alterations in intestinal microbiota correlate with susceptibility to type 1 diabetes. Diabetes. 2015;64(10):3510-20.

6. Belkaid Y, Hand TW. Role of the microbiota in immunity and inflammation. Cell. 2014;157(1):121-41.

7. Koenig JE, Spor A, Scalfone N, Fricker AD, Stombaugh J, Knight R, et al. Succession of microbial consortia in the developing infant gut microbiome. Proc Natl Acad Sci USA. 2011;108(Suppl 1):4578-85.

8. Dominguez-Bello MG, Costello EK, Contreras M, Magris M, Hidalgo G, Fierer N, et al. Delivery mode shapes the acquisition and structure of the initial microbiota across multiple body habitats in newborns. Proc Natl Acad Sci USA. 2010;107(26):11971-5.

9. Palmer C, Bik EM, DiGiulio DB, Relman DA, Brown PO. Development of the human infant intestinal microbiota. PLoS Biol. 2007;5(7):e177.

10. Hildebrandt MA, Hoffmann C, Sherrill-Mix SA, Keilbaugh SA, Hamady $M$, Chen $Y Y$, et al. High-fat diet determines the composition of the murine gut microbiome independently of obesity. Gastroenterology. 2009:137(5):1716-24.

11. Turnbaugh PJ, Backhed F, Fulton L, Gordon Jl. Diet-induced obesity is linked to marked but reversible alterations in the mouse distal gut microbiome. Cell Host Microbe. 2008;3(4):213-23.

12. Turnbaugh PJ, Ley RE, Mahowald MA, Magrini V, Mardis ER, Gordon JI. An obesity-associated gut microbiome with increased capacity for energy harvest. Nature. 2006;444(7122):1027-31.

13. Turnbaugh PJ, Ridaura VK, Faith JJ, Rey FE, Knight R, Gordon JI. The effect of diet on the human gut microbiome: a metagenomic analysis in humanized gnotobiotic mice. Sci Transl Med. 2009;1 (6):6ra14

14. Blaser MJ, Falkow S. What are the consequences of the disappearing human microbiota? Nat Rev Microbiol. 2009;7(12):887-94.

15. Faith JJ, Guruge JL, Charbonneau M, Subramanian S, Seedorf H, Goodman $\mathrm{AL}$, et al. The long-term stability of the human gut microbiota. Science. 2013;341(6141):1237439.
16. Knip $M$, Siljander $H$. The role of the intestinal microbiota in type 1 diabetes mellitus. Nat Rev Endocrinol. 2016;12(3):154-67.

17. Cox LM, Yamanishi S, Sohn J, Alekseyenko AV, Leung JM, Cho I, et al. Altering the intestinal microbiota during a critical developmental window has lasting metabolic consequences. Cell. 2014;158(4):705-21.

18. Qi C-J, Zhang Q, Yu M, Xu J-P, Zheng J, Wang T, et al. Imbalance of fecal microbiota at newly diagnosed type 1 diabetes in chinese children. Chin Med J. 2016;129(11):1298-304.

19. Valdes AM, Walter J, Segal E, Spector TD. Role of the gut microbiota in nutrition and health. BMJ. 2018;361:k2179.

20. Parekh PJ, Balart LA, Johnson DA. The influence of the gut microbiome on obesity, metabolic syndrome and gastrointestinal disease. Clin Transl Gastroenterol. 2015;6(6):e91.

21. Traversi D, Rabbone I, Ignaccolo MG, Carletto G, Racca I, Vallini C, et al. Gut microbiota diversity and T1DM onset: preliminary data of a case-control study. Hum Microbiome J. 2017:5:11-3.

22. Moher D, Liberati A, Tetzlaff J, Altman DG. Preferred reporting items for systematic reviews and meta-analyses: the PRISMA statement. Ann Intern Med. 2009;151(4):264-9.

23. Brown CT, Davis-Richardson AG, Giongo A, Gano KA, Crabb DB, Mukherjee N, et al. Gut microbiome metagenomics analysis suggests a functional model for the development of autoimmunity for type 1 diabetes. PLoS ONE. 2011;6(10):e25792.

24. de Goffau MC, Fuentes S, van den Bogert B, Honkanen H, de Vos WM, Welling GW, et al. Aberrant gut microbiota composition at the onset of type 1 diabetes in young children. Diabetologia. 2014;57(8):1569-77.

25. Maffeis C, Martina A, Corradi M, Quarella S, Nori N, Torriani S, et al. Association between intestinal permeability and faecal microbiota composition in Italian children with beta cell autoimmunity at risk for type 1 diabetes. Diabetes Metab Res Rev. 2016;32(7):700-9.

26. Murri M, Leiva I, Gomez-Zumaquero JM, Tinahones FJ, Cardona F, Soriguer F, et al. Gut microbiota in children with type 1 diabetes differs from that in healthy children: a case-control study. BMC Med. 2013;11(1):1-12.

27. Pellegrini S, Sordi V, Bolla AM, Saita D, Ferrarese R, Canducci F, et al. Duodenal mucosa of patients with type 1 diabetes shows distinctive inflammatory profile and microbiota. J Clin Endocrinol Metab. 2017;102(5):1468-77.

28. Soyucen E, Gulcan A, Aktuglu-Zeybek AC, Onal H, Kiykim E, Aydin A. Differences in the gut microbiota of healthy children and those with type 1 diabetes. Pediatr Int. 2014;56(3):336-43.

29. Stewart CJ, Nelson A, Campbell MD, Walker M, Stevenson EJ, Shaw JA, et al. Gut microbiota of type 1 diabetes patients with good glycaemic control and high physical fitness is similar to people without diabetes: an observational study. Diabet Med. 2017;34(1):127-34.

30. Mejia-Leon M, Petrosino J, Ajami N, Domínguez-Bello M, Calderon de la Barca A. HLA DQ/DR prevalence and microbiota disturbance in northwestern Mexican children with type 1 diabetes (1118.3). FASEB J. 2014;28(1_supplement):1118.3.

31. Giongo A, Gano KA, Crabb DB, Mukherjee N, Novelo LL, Casella G, et al. Toward defining the autoimmune microbiome for type 1 diabetes. ISME J. 2011;5(1):82-91.

32. Huang Y, Li SC, Hu J, Ruan HB, Guo HM, Zhang HH, et al. Gut microbiota profiling in Han Chinese with type 1 diabetes. Diabetes Res Clin Pract. 2018;141:256-63.

33. Leiva-Gea I, Sanchez-Alcoholado L, Martin-Tejedor B, Castellano-Castillo D, Moreno-Indias I, Urda-Cardona A, et al. Gut microbiota differs in composition and functionality between children with type 1 diabetes and MODY2 and healthy control subjects: a case-control study. Diabetes Care. 2018:41(11):2385-95.

34. Higuchi BS, Rodrigues N, Gonzaga MI, Paiolo JCC, Stefanutto N, Omori $W P$, et al. Intestinal dysbiosis in autoimmune diabetes is correlated with poor glycemic control and increased interleukin-6: a pilot study. Front Immunol. 2018;9:1689.

35. Salamon D, Sroka-Oleksiak A, Kapusta P, Szopa M, Mrozinska S, LudwigSlomczynska AH, et al. Characteristics of gut microbiota in adult patients with type 1 and type 2 diabetes based on nextgeneration sequencing of the 16S rRNA gene fragment. Pol Arch Intern Med. 2018;128(6):336-43.

36. Cinek O, Kramna L, Mazankova K, Odeh R, Alassaf A, Ibekwe MU, et al. The bacteriome at the onset of type 1 diabetes: a study from four geographically distant African and Asian countries. Diabetes Res Clin Pract. 2018;144:51-62. 
37. Davis-Richardson AG, Ardissone AN, Dias R, Simell V, Leonard MT, Kemppainen $\mathrm{KM}$, et al. Bacteroides dorei dominates gut microbiome prior to autoimmunity in Finnish children at high risk for type 1 diabetes. Front Microbiol. 2014;5:678.

38. Endesfelder D, Engel M, Zu Castell W. Gut immunity and type 1 diabetes: a mélange of microbes, diet, and host interactions? Curr Diabetes Rep. 2016;16(7):60

39. Kostic AD, Gevers D, Siljander H, Vatanen T, Hyotylainen T, Hamalainen $A M$, et al. The dynamics of the human infant gut microbiome in development and in progression toward type 1 diabetes. Cell Host Microbe. 2015;17(2):260-73.

40. Pinto E, Anselmo M, Calha M, Bottrill A, Duarte I, Andrew PW, et al. The intestinal proteome of diabetic and control children is enriched with different microbial and host proteins. Microbiology. 2017;163(2):161-74.

41. Rozanova GN, Voevodin DA, Stenina MA, Kushnareva MV. Pathogenetic role of dysbacteriosis in the development of complications of type 1 diabetes mellitus in children. Bull Exp Biol Med. 2002;133(2):164-6.

42. Gao X, Huynh B-T, Guillemot D, Glaser P, Opatowski L. Inference of significant microbial interactions from longitudinal metagenomics data. Front Microbiol. 2018:9:2319.

43. Gavin PG, Mullaney JA, Loo D, Cao KL, Gottlieb PA, Hill MM, et al. Intestinal metaproteomics reveals host-microbiota interactions in subjects at risk for type 1 diabetes. Diabetes Care. 2018;41(10):2178-86

44. Vatanen T, Franzosa EA, Schwager R, Tripathi S, Arthur TD, Vehik K, et al. The human gut microbiome in early-onset type 1 diabetes from the TEDDY study. Nature. 2018;562(7728):589-94.

45. Stewart CJ, Ajami NJ, O'Brien JL, Hutchinson DS, Smith DP, Wong MC, et al. Temporal development of the gut microbiome in early childhood from the TEDDY study. Nature. 2018;562(7728):583-8.

46. Endesfelder D, Zu Castell W, Ardissone A, Davis-Richardson AG, Achenbach $\mathrm{P}$, Hagen $\mathrm{M}$, et al. Compromised gut microbiota networks in children with anti-islet cell autoimmunity. Diabetes. 2014;63(6):2006-14.

47. Cole DK, Bulek AM, Dolton G, Schauenberg AJ, Szomolay B, Rittase W, et al. Hotspot autoimmune T cell receptor binding underlies pathogen and insulin peptide cross-reactivity. J Clin Invest. 2016;126(6):2191-204.

48. Hamer HM, Jonkers D, Venema K, Vanhoutvin S, Troost FJ, Brummer RJ. Review article: the role of butyrate on colonic function. Aliment Pharmacol Ther. 2008;27(2):104-19.
49. Furusawa Y, Obata Y, Fukuda S, Endo TA, Nakato G, Takahashi D, et al. Commensal microbe-derived butyrate induces the differentiation of colonic regulatory T cells. Nature. 2013;504(7480):446-50.

50. Brestoff JR, Artis D. Commensal bacteria at the interface of host metabolism and the immune system. Nat Immunol. 2013;14(7):676-84.

51. Wong JM, de Souza R, Kendall CW, Emam A, Jenkins DJ. Colonic health: fermentation and short chain fatty acids. J Clin Gastroenterol. 2006;40(3):235-43.

52. Arpaia N, Campbell C, Fan X, Dikiy S, van der Veeken J, deRoos $P$, et al. Metabolites produced by commensal bacteria promote peripheral regulatory T-cell generation. Nature. 2013;504:451.

53. Atarashi K, Tanoue T, Shima T, Imaoka A, Kuwahara T, Momose Y, et al. Induction of colonic regulatory $T$ cells by indigenous clostridium species. Science. 2011;331(6015):337-41.

54. Izcue A, Coombes JL, Powrie F. Regulatory lymphocytes and intestinal inflammation. Annu Rev Immunol. 2009;27:313-38.

55. Smith PM, Howitt MR, Panikov N, Michaud M, Gallini CA, Bohlooly YM, et al. The microbial metabolites, short-chain fatty acids, regulate colonic Treg cell homeostasis. Science. 2013;341(6145):569-73.

56. Bron PA, van Baarlen $P$, Kleerebezem M. Emerging molecular insights into the interaction between probiotics and the host intestinal mucosa. Nat Rev Microbiol. 2011;10:66.

57. Smits HH, Engering A, van der Kleij D, de Jong EC, Schipper K, van Capel $\mathrm{TM}$, et al. Selective probiotic bacteria induce IL-10-producing regulatory $T$ cells in vitro by modulating dendritic cell function through dendritic cellspecific intercellular adhesion molecule 3-grabbing nonintegrin. J Allergy Clin Immunol. 2005;115(6):1260-7.

58. Penders J, Thijs C, Vink C, Stelma FF, Snijders B, Kummeling I, et al. Factors influencing the composition of the intestinal microbiota in early infancy. Pediatrics. 2006;118(2):511-21.

59. Garcia E, Lopez R. Streptococcus pneumoniae type 3 encodes a protein highly similar to the human glutamate decarboxylase (GAD65). FEMS Microbiol Lett. 1995;133(1-2):113-8.

\section{Publisher's Note}

Springer Nature remains neutral with regard to jurisdictional claims in published maps and institutional affiliations.
Ready to submit your research? Choose BMC and benefit from:

- fast, convenient online submission

- thorough peer review by experienced researchers in your field

- rapid publication on acceptance

- support for research data, including large and complex data types

- gold Open Access which fosters wider collaboration and increased citations

- maximum visibility for your research: over $100 \mathrm{M}$ website views per year

At BMC, research is always in progress.

Learn more biomedcentral.com/submissions 